\title{
AVALIAÇÃO DAS CONDIÇÕES BUCAIS DE PACIENTES OBESOS
}

\author{
Camila Farias Santos ${ }^{a}$ \\ Rosane Borges Dias ${ }^{b}$ \\ Roberta Catapano Naves ${ }^{\mathrm{C}}$ \\ Andrea Nóbrega Cavalcanti ${ }^{d}$ \\ Érica Del Peloso Ribeiro ${ }^{\text {e }}$
}

\begin{abstract}
Resumo
A prevalência da obesidade tem aumentado nas últimas décadas, tornando-se uma preocupação para a saúde pública, pois esta condição é fator de risco para o desenvolvimento de várias doenças sistêmicas. Evidências científicas tem demonstrado que a obesidade também pode estar relacionada com problemas bucais como a cárie dentária e a doença periodontal. Uma vez que a obesidade pode influenciar a saúde bucal dos indivíduos, torna-se relevante para os profissionais da área de saúde buscar esclarecer a relação entre elas. Assim, o objetivo deste trabalho foi avaliar as condições bucais de indivíduos obesos. A amostra consistiu de 30 indivíduos obesos (IMC>30) não fumantes, sistemicamente saudáveis, que não receberam tratamento periodontal nos últimos 6 meses. O exame clínico periodontal completo, Índice CPO-D e o Índice de Massa Corporal (IMC) foram realizados. Foi feita também avaliação do hálito matinal dos indivíduos, por meio do Halimeter ${ }^{\circledR}$. A periodontite foi encontrada em $80 \%$ da população. A média do CPO-D foi de 14,73. Os índices de placa visível e gengival foram de $43,69 \%$ e $51,87 \%$, respectivamente. Diante desses resultados, fica clara a importância do profissional de Odontologia atuar como promotor de saúde para a população estudada.
\end{abstract}

Palavras-chave: Obesidade; Saúde bucal.

Autor correspondente: Rosane Borges Dias - rosanebd@gmail.com

a. Graduada em Odontologia pela Escola Bahiana de Medicina e Saúde Pública e Especialista em Periodontia (ABO-BA)

b. Graduada em Odontologia pela Escola Bahiana de Medicina e Saúde Pública, mestre e doutoranda em Patologia (UFBA/FIOCRUZ)

c. Mestrado em Estomatologia. Especialista em Periodontia (ABO-BA) e professora da Escola Bahiana de Medicina e Saúde Pública

d. Doutorado em Clínica Odontológica pela Universidade Estadual de Campinas. Professora da Escola Bahiana de Medicina e Saúde Pública

e. Doutorado em Clínica Odontológica (Área de concentração em Periodontia) pela Faculdade de Odontologia de Piracicaba (UNICAMP). Professora Adjunta da Escola Bahiana de Medicina e Saúde Pública e da Faculdade de Odontologia da Universidade Federal da Bahia 


\title{
EVALUATION OF ORAL CONDITIONS IN OBESE PATIENTS
}

\begin{abstract}
The prevalence of obesity has increased in recent decades, becoming a public health concern because this condition is a risk factor for the development of several systemic diseases. It can also be related to oral conditions such as dental caries and periodontal disease. Since obesity may influence the oral health of individuals, seeking to clarify the relationship between them is of great interest to health professionals. The objective of this study was to evaluate the oral conditions of obese individuals. The sample consisted of 30 obese non-smokers, systemically healthy, who had not received periodontal treatment within the last 6 months or used antibiotics and / or inflammatory in the last 3 months. The complete periodontal clinical examination, DMFT Index and Body Mass Index (BMI) were performed. The morning breath of the subjects was also made using the Halimeter $囚$. Periodontitis was found in $80 \%$, of the population, the mean DMFT was 14.73 , the average visible plaque index, gingival index were $43.69 \%$ and $51,87 \%$, respectively. Given these results, it is clear the importance of dental professional should work as a promoter of health for the population studied.

Keywords: Obesity; Oral health.
\end{abstract}

\section{INTRODUÇÃO}

A Organização Mundial de Saúde (OMS) define a obesidade como uma condição de acúmulo excessivo de gordura corporal em um determinado grau, onde a saúde e o bem estar dos indivíduos podem ser prejudicialmente afetados. Esta condição alcançou proporções epidêmicas globais, onde 300 milhões apresentam obesidade clínica em todas as faixas etárias e grupos socioeconômicos. ${ }^{(1)}$

A obesidade é um importante fator de risco para o desenvolvimento de doenças sistêmicas como o diabetes mellitus tipo II, hiperlipidemia, hipertensão, doenças cardiovasculares, colelitíase e alguns tipos de câncer. ${ }^{(1)}$ Ela também pode estar relacionada a diferentes problemas bucais como cárie dentária, doença periodontal, halitose e alteração do fluxo salivar. ${ }^{(2)}$

A relação entre a cárie dentária e a ingestão de alimentos está bem demonstrada na literatura científica, em virtude do papel dos açúcares na sua etiologia. Os hábitos alimentares relacionados à obesidade podem também determinar uma prevalência de cárie dentária, sendo um fator de risco comum para ambas as doenças, uma vez que tanto a quantidade de sacarose ingerida quanto a frequência de ingestão são importantes fatores envolvidos na etiologia da cárie e da obesidade. Como estas doenças apresentam fatores etiológicos em comum, obesos adultos podem ter um número maior de cáries dentárias do que indivíduos considerados dentro de padrões normais de peso.(3)

A etiologia da doença periodontal envolve dois grupos de fatores: o hospedeiro susceptível e a presença de bactérias patogênicas. ${ }^{(4)} \mathrm{O}$ processo de doença se desenvolve quando há uma quebra do equilíbrio existente entre a resposta do hospedeiro e o desafio microbiano. ${ }^{(4)}$ Neste contexto, o biofilme dental tem papel fundamental e é considerado o fator etiológico primário da doença periodontal. 
Este atua por meio de mecanismos diretos, causando destruição tecidual pela liberação de enzimas líticas e produtos citotóxicos; e indiretos, desencadeando as reações de defesa do hospedeiro que podem resultar em destruição progressiva do periodonto. ${ }^{(5)}$

Recentemente, a possibilidade de uma associação entre a doença periodontal e a obesidade tem sido estudada por alguns autores. ${ }^{(6-11)} \mathrm{A}$ plausibilidade biológica que explica a associação entre a obesidade e a periodontite está relacionada ao processo imuno-inflamatório, sendo que, o tecido adiposo libera citocinas pró-inflamatórias proporcionais à massa corporal do indivíduo. ${ }^{(12,13)}$ Esses mediadores inflamatórios como fator de necrose tumoral alfa, interleucina-1 beta e a interleucina-6, secretados em maior quantidade em pacientes obesos, podem levar a um estado hiperinflamatório, aumentando o risco de desenvolvimento da doença periodontal ou a sua progressão. ${ }^{(13)}$

Uma vez que a obesidade é uma condição cada vez mais frequente na sociedade moderna e pode influenciar na saúde bucal dos indivíduos, buscar esclarecer a relação entre elas é muito interessante para os profissionais da área de saúde. Diante disso, o objetivo deste trabalho foi descrever as condições bucais de indivíduos com obesidade.

\section{METODOLOGIA}

\section{DESENHO DO ESTUDO \\ E SELEÇÃO DA AMOSTRA}

Foi desenvolvido um estudo observacional do tipo transversal no Ambulatório Docente Assistencial da Bahiana (ADAB) - Unidade Acadêmica Cabula do Curso de Odontologia da Escola Bahiana de Medicina e Saúde Pública (EBMSP). Este estudo faz parte do projeto de pesquisa "Avaliação da relação entre a doença periodontal e a obesidade", aprovado pelo Comitê de Ética em Pesquisa da EBMSP (CEP/EBMSP), sob o protocolo 87/2009.
A participação dos indivíduos nesta pesquisa foi efetivada após a assinatura do Termo de Consentimento Livre e Esclarecido (Resolução 466/12).

A amostra foi composta por 30 indivíduos com idades entre 18 e 65 anos. Os participantes foram triados na Unidade de Triagem e Urgência (UTU/ EBMSP) no período de fevereiro a outubro de 2011. A seleção da amostra foi realizada segundo o critério de inclusão: ter pelo menos 10 dentes permanentes na boca, excluindo os terceiros molares. Os critérios de exclusão foram: realização de tratamento periodontal nos últimos 6 meses; pacientes portadores de aparelho ortodôntico; uso de medicamentos que pudessem interferir na condição periodontal (antibiótico e/ou anti-inflamatório) nos últimos 3 meses; tabagismo; pacientes portadores de diabetes mellitus ou outra condição sistêmica que pudesse interferir no curso da doença periodontal, como osteoporose; gestante ou lactante.

\section{ENTREVISTA}

Os participantes foram submetidos a uma entrevista sobre dados pessoais, história médica e odontológica. Para entrevistá-los foi usado um roteiro estruturado, que consistia de uma anamnese com uma série de perguntas sequenciadas, o qual foi a eles aplicado antes do exame periodontal. Um examinador treinado conduziu as entrevistas e preencheu os roteiros.

\section{AVALIAÇÃO DO IMC}

O índice de massa corporal (IMC) é definido pela divisão do peso (em quilogramas) pelo quadrado da altura em metros. (1) $A$ altura dos indivíduos foi mensurada em centímetros por meio de uma fita métrica instalada verticalmente na parede. $O$ peso foi aferido em quilogramas por uma balança digital (G-Life $®$, São Paulo, SP, Brasil), com graduação de 100 gramas e capacidade de $180 \mathrm{Kg}$. Suas categorias podem ser definidas da seguinte forma: abaixo do peso [IMC abaixo de 18,5], peso normal [IMC entre 18,5 e 24,9 ], sobrepeso [IMC entre 25,0 
e 29,9], obesidade grau I [IMC entre 30,0 e 34,9], obesidade grau II [IMC entre 35, O e 39,9] e obesidade grau III [IMC acima de 40,0].(1)

A medida do IMC foi realizada por um único examinador padronizado previamente.

\section{AVALIAÇÃO DAS CONDIÇÕES BUCAIS}

As condições bucais dos indivíduos foram avaliadas pela história odontológica, obtida através do roteiro, realizado previamente ao exame periodontal. A higiene oral foi avaliada através da freqüência de escovação e uso do fio dental, a história de cárie dentária através da realização de tratamento restaurador e a perda dentária através da realização de exodontia.

\section{ÍNDICE CPO-D}

Proposto por Klein \& Palmer, ${ }^{(14)}$ é o índice mais utilizado em levantamentos epidemiológicos. É objetivo e simples, apresentando características favoráveis que determinaram sua utilização universal nesses anos.

Em 1997, a OMS fez uma adequação do modelo inicial proposto por Klein \& Palmer, ${ }^{(14)}$ realizando alterações no critério de diagnóstico e códigos como mostra o Quadro 1.

Quadro 1 - Critérios e códigos propostos pela OMS para cálculo do CPO-D

\begin{tabular}{l|c}
\hline \multicolumn{1}{c|}{ CONDIÇÃo DENTAL } & Código \\
\hline Hígido & O \\
\hline Cariada & 1 \\
\hline Restaurada e cariada & 2 \\
\hline Restauração sem cárie & 3 \\
\hline Dente perdido devido a cárie & 4 \\
\hline Dente permanente perdido por outra razão que não seja a cárie & 5 \\
\hline Selante de fissura & 6 \\
\hline Apoio de ponte ou coroa protética & 7 \\
\hline Dentes não erupcionados & 8 \\
\hline Trauma/fratura & T \\
\hline Não informado & 9 \\
\hline
\end{tabular}

Fonte: OMS, 1997.

\section{AVALIAÇÃO PERIODONTAL}

O exame clínico periodontal foi realizado em todas as unidades dentárias permanentes, excluindo os terceiros molares. As unidades foram examinadas em seis sítios (mésio-vestibular, vestibular, disto-vestibular, mésio-lingual, lingual, disto-lingual). Este exame foi realizado com uma sonda periodontal milimetrada do tipo Carolina do Norte (PS$15 \mathrm{~mm}$ - Hu Friedy $®$, Chicago, IL). Neste exame, os parâmetros clínicos periodontais avaliados foram:
Índice de Placa, Índice Gengival, Profundidade de Sondagem, Sangramento a Sondagem, Posição da Margem Gengival e Nível de Inserção Clínica (NIC).

Todo exame clínico foi realizado por uma única examinadora calibrada. Esta foi submetida à calibração, sendo os resultados avaliados pelo coeficiente de correlação intra-classe (ICC). O ICC obtido foi de 0,87 para a medição do NIC. 
Em relação ao diagnóstico da doença periodontal, os indivíduos classificados com periodontite, foram aqueles com pelo menos 4 dentes apresentando 1 ou mais sítios com profundidade de sondagem $\geq 4 \mathrm{~mm}$ e nível de inserção clínica $>3 \mathrm{~mm} \cdot{ }^{(15)}$

\section{AVALIAÇÃO DOS COMPOSTOS SULFURADOS VOLÁTEIS}

Essa avaliação foi feita no período da manhã com os pacientes em jejum e sem escovar os dentes. Foi solicitado também que os pacientes evitassem cosméticos e perfumes no dia das medidas do hálito, de acordo com a proposta de Kozlovsky et al. ${ }^{(16)}$ Medidas quantitativas dos CSV foram realizadas com a utilização de um monitor de sulfetos portátil (Halimeter $\mathbb{R}$ RH-17E, Interscan, Simi Valley, CA, EUA). O pico máximo de compostos sulfurados voláteis foi determinado em partes por bilhão de componentes sulfidrílicos pela leitura direta da escala analógica do monitor.

\section{ANÁLISE DOS DADOS}

Inicialmente, realizou-se uma análise descritiva das variáveis estudadas obtendo-se as frequências simples e relativas para todas as variáveis. Em seguida, todas as variáveis foram transformadas em dicotômicas, de acordo com a distribuição que apresentaram. As médias das variáveis contínuas: idade, IMC, IP, IC e medida do hálito foram obtidas através do Programa Bioestat ${ }^{\circledR}$ (versão 5.3).

\section{RESULTADOS}

Dos 30 pacientes obesos examinados, 4 (13,3\%) indivíduos eram do sexo masculino e $26(86,6 \%)$ do sexo feminino. $A$ idade destes pacientes variou entre 18 a 65 anos, com uma média de 38,99 anos. Os dados em relação aos aspectos sociais e comportamentais desta amostra podem ser observados na Tabela 1.

Tabela 1 - Características sócio-demográficas e de saúde geral da população estudada

\begin{tabular}{ll}
\hline VARIÁVEIS & No $(\%)$ \\
\hline Sexo & \\
$\quad$ Feminino & $26(86,6)$ \\
$\quad$ Masculino & $\mathrm{O}(13,4)$ \\
Escolaridade & \\
$\quad$ Ensino Fundamental & $\mathrm{O}(30)$ \\
$\quad$ Ensino Médio & $19(63,3)$ \\
$\quad$ Ensino Superior & $\mathrm{O}(6,7)$ \\
Renda Salarial & \\
$\quad$ Menor ou igual a um salário mínimo & $22(73,3)$ \\
Maior que um salário mínimo & $\mathrm{O}(26,7)$ \\
Hipertensão arterial & \\
$\quad$ Ausente & $22(73,3)$ \\
Presente & $\mathrm{O}(26,7)$ \\
Prática de atividade física & \\
$\quad$ Ausente & $22(73,4)$ \\
Presente & $\mathrm{O}(26,6)$ \\
Uso de contraceptivo oral/Reposição hormonal* & \\
$\quad$ Ausente & $26(86,6)$ \\
Presente & $\mathrm{O}(13,4)$ \\
Uso de medicamento & $19(63,4)$ \\
$\quad$ Ausente & $11(36,6)$ \\
Presente &
\end{tabular}


O índice CPO-D encontrado na população estudada foi de 14,73. As características comportamen- tais em relação aos hábitos de higiene e de saúde bucal dos pacientes estão descritos na Tabela 2.

Tabela 2 - Características comportamentais e de saúde bucal da população estudada

\begin{tabular}{lc}
\hline \multicolumn{1}{c}{ VARIÁVEIS } & No $(\%)$ \\
\hline Freqüência de escovação & \\
Nenhuma/uma vez ao dia & $\mathrm{O}(6,6)$ \\
Duas ou mais vezes ao dia & $28(93,4)$ \\
Uso de fio dental & \\
$\quad$ Sim & $\mathrm{O}(26,7)$ \\
Não & $22(73,3)$ \\
Uso de enxaguatório & $10(33,4)$ \\
Sim & $20(66,6)$ \\
Não & \\
Freqüência de visita ao dentista & $21(70)$ \\
Uma vez/ano & $09(30)$ \\
Duas ou mais vezes/ano & \\
Realização de Tratamento restaurador & $22(73,3)$ \\
Sim & $O 8(26,7)$ \\
Não & \\
Realização de Raspagem e Alisamento Radicular & $11(36,7)$ \\
Sim & $19(63,3)$ \\
Não & \\
Realização de Exodontia & $23(76,6)$ \\
Sim & $07(23,4)$ \\
Não &
\end{tabular}

Fonte: BAHIANA, 2011.

Em relação a obesidade, 21 (70\%) pacientes apresentaram Grau I, 3 (10\%) pacientes Grau II e 6 (20\%) Grau III . A periodontite foi observada em 24
(80\%) dos pacientes. A classificação da doença periodontal de acordo com o grau de obesidade está descrita na Tabela 3.

Tabela 3 - Classificação da doença periodontal segundo o grau de obesidade da população estudada

\begin{tabular}{cccc}
\hline Diagnóstico Periodontal & $\begin{array}{c}\text { Obesidade Grau I } \\
\mathrm{n}(\%)\end{array}$ & $\begin{array}{c}\text { Obesidade Grau II } \\
\mathrm{n}(\%)\end{array}$ & $\begin{array}{c}\text { Obesidade Grau III } \\
\mathrm{n}(\%)\end{array}$ \\
\hline Saúde & $3(10)$ & $1(3,33)$ & $2(6,67)$ \\
Periodontite Leve & $2(6,67)$ & 0 & $3(10)$ \\
Periodontite Moderada & $9(30)$ & $2(6,67)$ & 0 \\
Periodontite Severa & $7(23,33)$ & 0 & $1(3,33)$ \\
\hline
\end{tabular}

Fonte: BAHIANA, 2011.

Observou-se que os pacientes com periodontite apresentaram médias maiores relacionadas às variáveis: índice de placa visível, índice gengival e avaliação do hálito (Tabela 4). 
Tabela 4 - Média e desvio padrão das variáveis contínuas dos grupos com e sem periodontite da população estudada

\begin{tabular}{|c|c|c|c|c|}
\hline \multirow{2}{*}{ VARIÁVEIS } & \multicolumn{2}{|c|}{$\begin{array}{l}\text { SEM DOENÇA PERIODONTAL } \\
\qquad(\mathrm{N}=6)\end{array}$} & \multicolumn{2}{|c|}{$\begin{array}{c}\text { COM DOENçA PERIODONTAL } \\
\qquad(\mathrm{N}=24)\end{array}$} \\
\hline & MÉDIA & Dp & MÉDIA & Dp \\
\hline IDADE & 34,66 & 4,55 & 41,79 & 11,17 \\
\hline IMC & 38,16 & 6,82 & 35,24 & 6,28 \\
\hline ÍNDICE DE PLACA VISIIVEL & 23,83 & 7,86 & 47,36 & 14,59 \\
\hline ÍNDICE GENGIVAL & 26,77 & 9,32 & 57,46 & 14,95 \\
\hline HALIMETER & 107,128 & 12,01 & 279,13 & 212,31 \\
\hline
\end{tabular}

Fonte: BAHIANA, 2011.

\section{DISCUSSÃO}

A obesidade é considerada um problema para a saúde pública, pois esta condição é fator de risco para o desenvolvimento de várias doenças sistêmicas. ${ }^{(1)}$

A obesidade é caracterizada por um estado inflamatório crônico, que pode agravar condições inflamatórias preexistentes como a doença periodontal. (17) Atualmente, existe um consenso na literatura de que o tecido adiposo não é um tecido estático biologicamente. As adipocinas, moléculas bioativas, secretadas pelo tecido adiposo, são capazes de induzir a produção e a secreção de algumas substâncias no organismo, como citocinas (TNF- $\alpha$, IL-6), hormônios que se ligam as proteínas (leptina e adiponectina), proteínas hemostáticas (Inibidor-1 de Ativador de Plasminogênio), reguladores de pressão sanguínea (angiotensinogênio), promotores de angiogênese (fator de crescimento vascular endotelial) e elementos da fase aguda (proteína C- reativa). ${ }^{(13)}$

A média de idade dos indivíduos deste estudo foi de $\pm 38,99$ anos. Dentro deste contexto, dados do Ministério da Saúde ${ }^{(18)}$ demonstraram que a maior frequência de obesidade em homens ocorre entre 45 e 54 anos de idade. Nessa faixa etária, o índice aumenta mais de três vezes comparado àqueles entre 18 e 24 anos, diminuindo nas faixas seguintes. Por outro lado, nas mulheres, a frequência aumenta mais de seis vezes entre os períodos de 18 e 24 anos e de 55 a 64 anos, e diminui a partir dos 65 anos.
Um das limitações do presente estudo foi a utilização do IMC como medida para avaliar o excesso de gordura corpórea. Apesar deste método ser utilizado em grande escala nos estudos epidemiológicos e ser preconizado pela Organização Mundial de Saúde (OMS), possui algumas limitações. ${ }^{(1)}$ Este índice não faz distinção entre massa adiposa e massa magra, não identificando a localização da massa gordurosa no organismo. ${ }^{(19)}$ Desta maneira, faz-se necessário a associação do IMC com outras medidas como circunferência abdominal, biopedância, relação da cintura-quadril. ${ }^{(20)}$

Em relação ao grau de obesidade proposto pela OMS, observou-se que a maioria dos indivíduos deste estudo apresentava Grau I de obesidade. Contudo, a obesidade mórbida foi observada em $20 \%$ dos indivíduos. O tratamento mais eficaz para esta condição tem sido a cirurgia bariátrica, conhecida também como cirurgia de redução de estômago. ${ }^{(21)}$ Alguns trabalhos tem demonstrado que os indivíduos após cirurgia bariátrica tem apresentado algumas alterações bucais como hipersensibilidade dentinária, cáries, doença periodontal e halitose. ${ }^{(22-24)} \mathrm{Em}$ relação a condição periodontal, Mariscano et al. (2011) $)^{(22)}$ e de Moura-Grec et al. $(2014)^{(24)}$ observaram uma maior prevalência de doença periodontal em indivíduos operados do que nos obesos. Há uma escassez de estudos que avaliem os efeitos da cirurgia bariátrica na condição 
periodontal, com isso, é necessário o desenvolvimento de estudos longitudinais com a finalidade de avaliar essa relação.

Os resultados deste estudo demonstraram que os indivíduos obesos com periodontite apresentavam um maior índice de placa visível e índice gengival, corroborando com os achados de Löe et al. $(1965)^{(25)}$ que demonstraram que o acúmulo de biofilme nas superfícies dos dentes é um fator de risco para o desenvolvimento da inflamação gengival. Diante disso, o presente estudo realizou o Índice de Placa Visível e o Índice Gengival com o objetivo de uma melhor avaliação deste fator etiológico.

Neste estudo, houve uma alta prevalência de indivíduos com periodontite. Este resultado pode estar relacionado com os hábitos comportamentais relacionados ao estilo de vida dos pacientes obesos, tais como: alimentação inadequada, não realização de atividade física e menores cuidados com saúde geral e bucal. ${ }^{(2)}$ Ainda, foi observado neste estudo que os indivíduos tinham hábitos de higiene oral deletérios, como uma escovação inadequada e o não uso do fio dental, podendo também explicar a alta prevalência da doença periodontal.

O exame periodontal, na maioria dos estudos epidemiológicos, é realizado utilizando o Índice das Necessidades de Tratamento Periodontal Comunitário (CPITN). Esse índice foi projetado, claramente, para avaliar grupos de comunidades populosas, a fim de determinar as necessidades de tratamento e facilitar as estratégias profiláticas e terapêuticas, e não para descrever a prevalência e a severidade da doença periodontal. ${ }^{(26)}$ Alguns estudos têm questionado a utilização do CPITN, como um estudo realizado por Baelum et al. (1995) que concluíram que as marcações do índice CPITN não apresentaram uma correlação coerente com os níveis de perda de inserção, mas tendem a superestimar a prevalência e a severidade entre indivíduos mais jovens, enquanto subestimam tais parâmetros em populações mais velhas. ${ }^{(27)}$ Contudo, nesta pesquisa, o exame periodontal completo foi realizado, associando-se dois parâmetros clínicos: profundidade de sondagem e nível de inserção clínica.
No Projeto Saúde Bucal - Brasil realizado nos anos de 2002/2003 pelo Ministério da Saúde, o CPO-D encontrado para indivíduos na faixa etária de 35 a 44 anos foi de 20,13 . $^{(28)}$ No presente estudo, o CPO-D foi de 14,73. Apesar de o valor ter sido alto, é inferior ao encontrado no SB-Brasil, isto pode ser justificado devido um maior acesso dos indivíduos deste estudo, pacientes da EBMSP, a cuidados odontológicos preventivos. A presença de cárie dentária nos indivíduos obesos do presente estudo pode estar relacionada com os hábitos comportamentais dos indivíduos obesos, como o alto consumo de açúcar e a falta de cuidado com os hábitos de higiene oral.

A halitose é caracterizada por um odor desagradável do ar exalado, tendo a gengivite e periodontite como principais causas. ${ }^{(29)}$ Nesse sentido, os resultados do presente estudo demonstraram que os indivíduos obesos com periodontite apresentavam uma halitose mais severa que os pacientes sem doença periodontal. As bolsas periodontais são consideradas como uma das principais fontes de compostos sulfurados voláteis devido às bactérias anaeróbias proteolíticas gram-negativas, ${ }^{(30)}$ isto explicaria a halitose mais severa nos pacientes com periodontite deste estudo. Além disso, os compostos sulfurados voláteis são capazes de alterar a permeabilidade do periodonto e a resposta inflamatória dos indivíduos com halitose. Em um estudo in vitro foi demonstrado que a metilmercaptana aumenta a secreção de prostaglandina $E_{2}$ e colagenase, o que justificaria uma resposta inflamatória mais exacerbada e consequentemente maior destruição tecidual.(31)

\section{CONCLUSÃO}

Pode-se concluir, com este estudo, que a alta prevalência de periodontite nos pacientes obesos torna-se uma preocupação para a saúde pública, pois eles estão mais susceptíveis a perda dentária e outras doenças associadas. Assim, o tratamento e manutenção das condições periodontais são necessárias para a promoção de saúde na população estudada. 


\section{REFERÊNCIAS}

1. World Health Organization. Obesity and overweight. Geneva; 2003.

2. Mathus-Vliegen EM, Nikkel D, Brand HS. Oral aspects of obesity. Int Dent J. 2007; 57:249-56.

3. Traebert, J, Moreira EAM, Bosco VL, Alemida ICS. Transição alimentar: problema comum à obesidade e à cárie dentária. Rev. nutr. 2004;17(2):247-53.

4. Quirynen M, Teughels W, De Soete M, van Steenberghe D. Topical antiseptics and antibiotics in the initial therapy of chronic adult periodontitis: microbiological aspects. Periodontol 2000. 2001; 28:72-90.

5. Page RC, Offenbacher S, Schroeder HE, Seymour GJ, Kornman KS. Advances in the pethogenesis of periodontitis: summary of developments, clinical implications and future directions. Periodontol 2000. 1997; 14:216-48.

6. Saito T, Shimazaki Y, Koga T, Tsuziki M, Ohshima A. Relationship between upper body obesity and periodontitis. J Dent Res. 2001; 28:1631-36.

7. Dalla Vechia CF, Susin C, Rosing CK, Oppermann RV, Albandar JM. Overweight and Obesity as risk indicators for Periodontitis in adults. J Periodontol. 2005; 76(10):1721-8.

8. Genco RJ, Grossi SG, Ho A, Nishimura F, Murayama Y. A proposed model linking inflammation to obesity, diabetes and periodontal infections. J Periodontol. 2005; 76(11):2075-84.

9. Ylostalo P, Suominentaipal EL, Reunanen A, Knuuttila M. Association between body weight and periodontal infection. J Clin Periodontol. 2008; 35:297-302.

10. Dias RB, Almeida MOS, Ribeiro EDP, Naves RC. Estudo da obesidade como indicador de risco para a doença periodontal. Revista Periodontia. 2011; 21(2):70-78.

11. Palle AR, Reddy CM, Shankar BS, Gelli V, Sudhakar J, Reddy KK. Association between obesity and chronic periodontitis: a cross sectional study. J Contemp Dent Pract. 2013 Mar 1;14(2):168-73.

12. Pischon N, Heng N, Bernimoulin JP, Kleber BM, Willich SN, Pischon T. Obesity, inflammation and
Periodontal disease. J. Dent. Res. 2007; 86: 400-409.

13. Ritchie CS. Obesity and periodontal disease. Periodontol 2000. 2007; 44: 154-163.

14. Klein H, Palmer CE. Dental caries in American Indian children. Public Health Bull 1937; 239:1-53.

15. Gomes Filho IS, Macedo TCN, Cruz SS, Soledade KR, Trindade SC, Sarmento VA. Comparação de critérios que determinam o diagnóstico clínico da doença periodontal. Rev odonto ciênc 2006; 21(51):77-81.

16. Kozlovsky A, Gordon D, Gelernter I, Loesche WJ, Rosenberg M. Correlation between the BANA test and oral malodor parameters. J Dent Res. 1994;73(5):1036-42.

17. Bezerra BB, Sallum EA, Sallum AWP. Obesity and periodontal disease: why suggest such relationship? An overview. Braz j. oral. sci. 2007; 6(23): 142O-1422.

18. Brasil. Ministério da Saúde. VIGITEL BRASIL 2010. [acesso em 17 jun. 2011]. Disponível em: http://saude.gov.br/bvs.

19. Gaio EJ. Efeito da obesidade na progressão da perda de inserção periodontal: estudo de Porto Alegre. [dissertação]. Porto Alegre: Universidade Federal do Rio Grande do Sul, 2008.

2O. Khander YS, Bawadi HÁ, Haroun TF, Alomari $M$, Tayyem RF. The association between periodontal disease and obesity among adults in Jordan. J Clin Periodontol 2008; 36:18-24.

21. Waseem, T.; Mogensen, K.M.; Lautz, D.B.; Robinson M.K. Pathophysiology of obesity: why surgery remains the most effective treatment. Obes surg. 2007; 17(10): 1389-98.

22. Marsicano JA, Grec PG, Belarmino LB, Ceneviva $\mathrm{R}$, Peres $\mathrm{SH}$. Interfaces between bariatric surgery and oral health:longitudinal survey. Acta cir. bras. 2011; 26 Suppl 2:79-83.

23. Dupim Souza AC, Franco CF, Pataro AL, Guerra T, de Oliveira Costa F, da Costa JE. Halitosis in obese patients and those undergoing bariatric surgery. Surg Obes Relat Dis. 2013;9(2):315-21.

24. de Moura-Grec PG, Yamashita JM, Marsicano JA, Ceneviva R, de Souza Leite CV, de Brito GB, et al. Impact of bariatric surgery on oral health conditions: 6-months cohort study. Int Dent J. 2014;64(3):144-9. 
25. Löe H, Theilade E, Jensen B. Experimental gingivitis in man. J Periodontol 1965; 36: 177-87.

26. Izumi A, Yoshihara A, Hirotomi T, Miyazaki $H$. The relationship between serum lipids and periodontitis in elderly non-smokers. J Periodontol 2009; 80:740-8.

27. Baelum V, Manji F, Wanzala P, Fejerskov O. Relationship between CPITN and periodontal attachment loss findings in an adult population. J Clin Periodontol 1995; 22:146-52.

28. Brasil. Ministério da Saúde. Projeto SB Brasil 2003. Condições de saúde bucal da população brasileira 2002-2003. Resultados principais. Brasília; 2003.

29. Lee PCC, Mak WY, Newsome P. The aetiology and treatment of oral halitosis: an update. Hong Kong Med J. 2004; 1O(6):414-18.

30. Morita M, Wang HL. Association between oral malodor and adult periodontitis: a review. J Clin Periodontol. 2001; 28(9): 813-9.

31. Ratkay JG, Waterfield JD, Tonzetich. Stimulation of enzyme and cytokine production by methyl mercaptan in human gingival fibroblast and monocyte cell cultures. Arch Oral Biol. 1995; 4O: 337-344. 